\title{
Discrepancies of Patellofemoral Indices between Supine and Standing Merchant Views
}

\author{
Tae-Hoon Kim, MD, Jong-Soo Lee, MD, and Kwang-Jun Oh, MD \\ Department of Orthopedic Surgery, Konkuk University School of Medicine, Seoul, Korea
}

\begin{abstract}
Purpose: This research was conducted to compare supine and standing Merchant views, to observe radiographic changes of the patellofemoral joint according to weight-bearing conditions, and to evaluate correlation factors affecting patellofemoral joint indices.

Materials and Methods: Forty-four patients without skeletal problems were selected for this study. Patellar tilt angle, lateral patellofemoral angle, congruence angle, lateral subluxation distance and lateral patellar displacement were measured on each radiograph and evaluated for statistical significance. Possible correlation factors that can affect the radiographic discrepancy were analyzed using the univariate and multivariate regression analysis.

Results: In the standing Merchant view, the patellar tilt angle, congruence angle and lateral patellar displacement were significantly decreased ( $<<0.001)$, whereas the lateral patellofemoral angle was significantly increased $(\mathrm{p}<0.001)$ compared to that in the supine Merchant view. Thigh width $(\mathrm{p}<0.001$, $\left.\mathrm{r}^{2}=0.22\right)$ and radiographic $\mathrm{Q}$-angle $\left(\mathrm{p}<0.001, \mathrm{r}^{2}=0.34\right)$ were found correlated with the radiographic discrepancy of congruence angle. Discrepancy of the lateral patella displacement increased as radiographic $\mathrm{Q}$-angle increased $\left(\mathrm{p}=0.027, \mathrm{r}^{2}=0.112\right)$.

Conclusions: Compared with the supine Merchant view, standing (weight-bearing) Merchant view showed decreased patella tilt angle, congruence angle, and lateral patella displacement. The results indicate that both supine and standing Merchant views should be considered in the radiographic evaluation of the patellofemoral joint.
\end{abstract}

Keywords: Patellofemoral joint, Supine, Standing, Merchant view, Patellofemoral index

\section{Introduction}

Patellar maltracking is diagnosed in approximately $50 \%$ of the patients with patellofemoral joint pain. It is characterized by excessive lateral translation of the patella relative to the femur and induction of pain in full knee extension ${ }^{1,2)}$. In most cases, patellar tracking is assessed in non-weight-bearing conditions where the patient is placed in the sitting position for clinical examination

Received August 5, 2013; Revised (1st) October 1, 2013;

(2nd) November 13, 2013; (3rd) December 21, 2013;

Accepted December 23, 2013

Correspondence to: Kwang-Jun Oh, MD

Department of Orthopedic Surgery, Konkuk University School of

Medicine, 263 Achasan-ro, Gwangjin-gu, Seoul 143-701, Korea

Tel: +82-2-2030-7615, Fax: +82-2-2030-7369

E-mail:damioh@gmail.com

This is an Open Access article distributed under the terms of the Creative Commons Attribution Non-Commercial License (http://creativecommons.org/licenses/by-nc/3.0/) which permits unrestricted non-commercial use, distribution, and reproduction in any medium, provided the original work is properly cited. and in the supine or sitting position with the knee in flexion for radiographic assessment, failing to replicate the patellofemoral joint motion during weight-bearing activities ${ }^{3-5)}$. As a result, the risk of misdiagnosis increases, and improper treatment or unnecessary surgery may be carried out based upon non-weightbearing assessments.

There is limited understanding of the overall kinematics of the patellofemoral joint under weight-bearing conditions. Patellofemoral joint alignment and motion were assessed in the supine position under non-weight-bearing conditions in most studies in the literature, except for a few reports ${ }^{6-9)}$. In addition, patellofemoral joint motion is also affected by quadriceps femoris con$\operatorname{traction}^{10)}$ and joint loading ${ }^{11,12)}$. Thus, we believe that thorough investigation on the influence of weight-bearing as opposed to non-weight-bearing is essential for comprehensive understanding of the patellofemoral joint.

In a recent research, Baldini et al. ${ }^{5)}$ identified radiographic changes from the supine Merchant (supine-M) view and standing weight-bearing Merchant (standing-M) view obtained after total knee arthroplasty: lateral tilt and subluxation of the patella 
were neutralized and reduced under weight-bearing, indicating the efficacy of weight-bearing radiography in patellofemoral joint assessment ${ }^{5}$. In this study, we hypothesized that weightbearing conditions would result in changes in the patellofemoral alignment, such as patellar tilt angle (TILT) and lateral patellar displacement (LAT-PD), in patients without patellofemoral joint pain and radiologic abnormalities other than the complaint of knee pain. In addition, we investigated factors that cause radiographic discrepancy between the two positions.

\section{Materials and Methods}

\section{Patient Selection and Radiographic Imaging}

Of the patients who visited our clinic with a major complaint of knee pain, 44 patients without patellofemoral joint pain and radiographic abnormalities were included in this study. There were 22 males and 22 females with a mean age of 27.8 years (range, 18 to 39 years). In all patients, supine- $M$ and standing- $M$ views were taken on the same occasion from the 44 left knees and 44 right knees. Weight-bearing full leg standing view was also obtained in all patients.

The supine- $M$ view was taken with the patient placed in the supine position maintaining $45^{\circ}$ of femorotibial angle and the $\mathrm{X}$ ray beam directed at $30^{\circ}$ angle from the horizon. The standing-M view was taken with the patient maintaining $45^{\circ}$ of flexion while standing and the X-ray beam directed vertical to the floor. The angle between the femur and tibia was maintained during the procedure by using an orthopaedic goniometer. Care was taken to ensure the reflected X-ray beam to have the same angle with respect to the patellofemoral joint on both the supine- $M$ and standing-M views (Fig. 1$)^{5}$.

\section{Radiographic Measurements}

The supine- $M$ and standing- $M$ views were obtained using a general method on the same occasion. The TILT (+angle: lateral tilt), patellofemoral angle (+angle: lateral divergence), congruence angle (Cong, +angle: increased incongruence), and LAT-PD (+angle: lateral displacement) were measured on each view and evaluated for statistical analysis. To analyze the influence of various factors on discrepancy in the supine- $M$ and standing- $M$ view measurements, possible influencing factors (gender, age, femoral neck offset [F-OFF], femoral length [FL], tibial length [TL], mechanical axis of the lower limb, thigh width [TW], calf width [CW], and radiographic Q-angle [Rad-Q]) were investigated.

Radiographic measurements for each parameter were performed twice by one investigator with an interval of one week using Picture Archiving Communication System (Infinitt, Seoul, Korea) and the mean values were used for analysis (Fig. 2).

The TILT was defined as the angle between a line connecting the two edges of the patella (corner-to-corner line) and the horizontal line. Increased TILT was associated more lateral patellar tilt, and conversely decreased TILT was related to more medial patellar tilt. The Cong was measured as the angle between a line that bisects the sulcus angle and a line that is drawn from the lowest point in the intercondylar groove through the articular ridge of the patella. If Cong was decreased, it meant congruence was increased. Patella lateral subluxation distance (SUB-D) was defined as the distance from the lowest point of the femoral trochlea to the median ridge of the patella: if the median ridge was located lateral to the lowest point of the trochlea, the distance was assigned a positive value, whereas if located medial, a negative value was given. Lateral patellofemoral angle (LAT-PF) was defined as the angle created by the intercondylar line of the femur

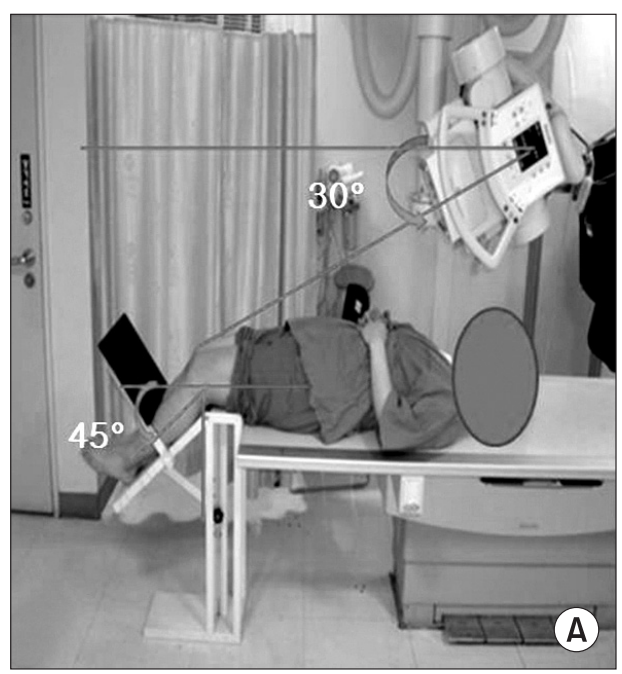

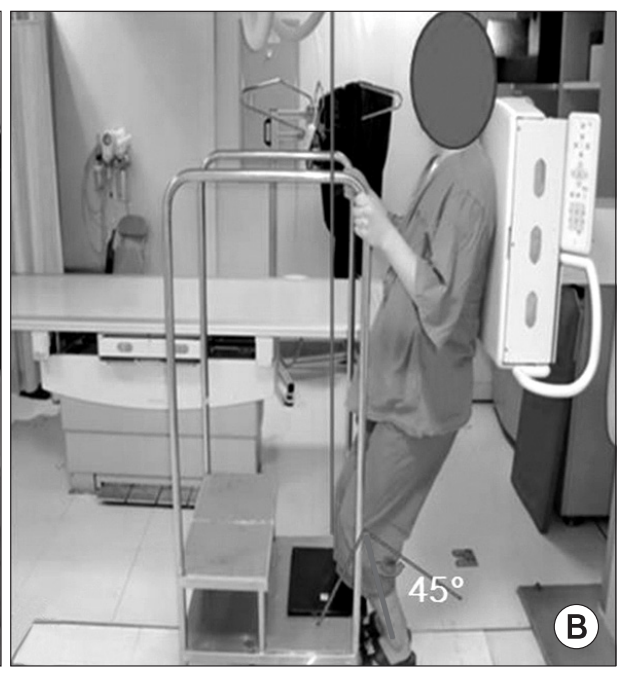

Fig. 1. Patient positioning for the supine, non-weight-bearing view (A) and standing, weight-bearing Merchant view (B). (B) The patient stands in a semi-squatting position with the knees in $45^{\circ}$ of flexion: a modified patient positioning technique for obtaining a weight-bearing Merchant view radiograph. 

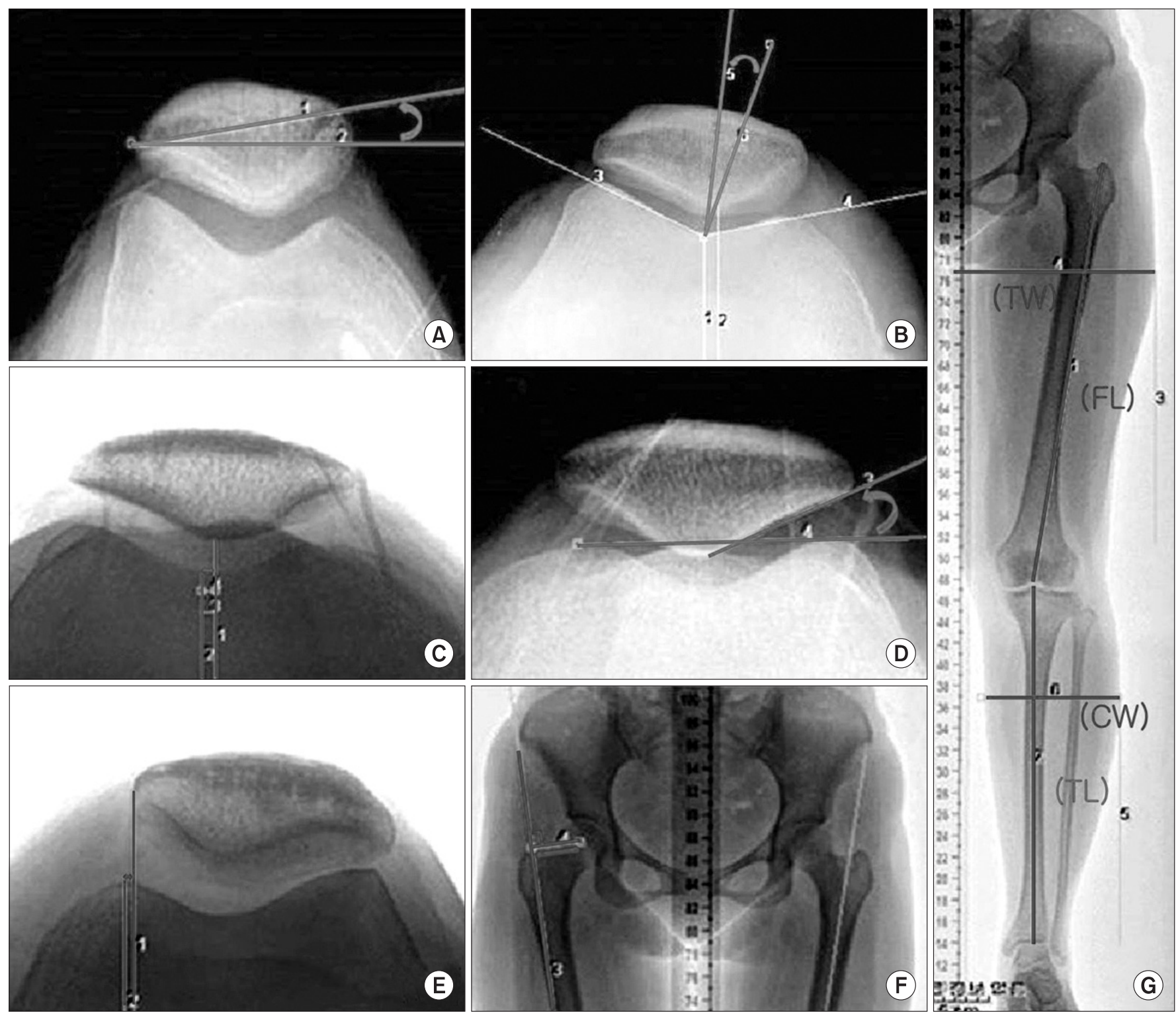

geat

Fig. 2. Definition of various angles, distances and lengths on the Merchant views and weight bearing full leg standing view. (A) Patellar tilt angle, (B) congruence angle, (C) patella lateral subluxation distance, (D) lateral patellofemoral angle, (E) lateral patellar displacement, (F) femoral offset, (G) TW: thigh width, FL: femoral length, CW: calf width, TL: tibial length.

and a line drawn parallel to the lateral articular surface of the patella. LAT-PD was measured as the distance from the medial end of the patella to the most anterior point of the medial condyle of the femur. A positive value was given if the medial end of the patella was located laterally, whereas a negative value was assigned for medial location ${ }^{13,14)}$.

Additional measurements were performed to investigate factors that could influence the values of the above-described five parameters measured from the supine- $M$ and the standing- $M$ views. The F-OFF was measured as the distance from the center of the rotation of the femoral head to the long axis of the femur.
The FL was measured from the center of the femoral intercondylar fossa through the long axis of the femur to the tip of the bone on the weight-bearing full leg standing view. Similarly, the TL was measured from the tibial intercondylar eminence through the long axis to the distal end of the tibia on the weight- bearing full leg standing view. The TW and CW were measured inward from the most protruded portion of the thigh and calf, respectively, on the weight-bearing full leg standing view. The Rad-Q was defined as the angle between a line extending from the tibial tubercle to the mid-patella and a line from the anterior superior iliac spine to the mid-patella on the weight-bearing full leg standing view. 


\section{Statistical Analysis}

PASW ver. 17.0 (SPSS Inc., Chicago, IL, USA) was used for statistical analysis. Intraobserver reliability was calculated using intraclass correlation coefficients (ICC). The intraobserver agreement percentage and kappa coefficient were $93 \%$ and 0.86 , respectively, and the lowest values of all the parameters were higher than those suggested by the ICC. Statistically significant differences between the values obtained from the supine- $M$ and standing- $\mathrm{M}$ views were investigated using paired t-test. Possible influencing factors including gender, age, F-OFF, FL, TL, mechanical axis of the lower limb, TW, CW, and Rad-Q were assessed using univariate and multivariate regression analysis. A $\mathrm{p}<0.05$ was considered statistically significant.

\section{Results}

The mean TILT was significantly decreased from $5.3^{\circ} \pm 2.5^{\circ}$ (range, $1.5^{\circ}$ to $9.8^{\circ}$ ) on the supine-M view to $3.7^{\circ} \pm 2.0^{\circ}$ (range, $0.1^{\circ}$ to $8.5^{\circ}$ ) on the standing- $\mathrm{M}$ view ( $\left.\mathrm{p}=0.002\right)$. The mean Cong was reduced from $-3.5^{\circ} \pm 4.5^{\circ}$ (range, $-11.0^{\circ}$ to $11.2^{\circ}$ ) on the supine$\mathrm{M}$ view to $-8.7^{\circ} \pm 2.4^{\circ}$ (range, $-12.0^{\circ}$ to $-2.8^{\circ}$ ) on the standing-M view $(\mathrm{p}<0.001)$, indicating increased congruence of the knee joint. The mean SUB-D was significantly shorter on the stand- ing- $M$ view $(-2.0 \pm 1.8 \mathrm{~mm}$; range, -5.8 to $4.6 \mathrm{~mm})$ than on the supine-M view ( $-1.0 \pm 2.8 \mathrm{~mm}$; range, -6.6 to $4.6 \mathrm{~mm})(\mathrm{p}=0.039)$. The mean LAT-PF was notably increased from $6.8^{\circ} \pm 3.8^{\circ}$ (range, $1.1^{\circ}$ to $15.8^{\circ}$ ) on the supine-M view to $11.3^{\circ} \pm 4.7^{\circ}$ (range, $1.0^{\circ}$ to $\left.20.9^{\circ}\right)$ on the standing-M view $(\mathrm{p}<0.001)$. The mean LAT-PD was significantly shorter on the standing-M view $(-3.5 \pm 3.7 \mathrm{~mm}$; range, -9.6 to $8.2 \mathrm{~mm})$ than on the supine- $M$ view $(-1.9 \pm 3.6$ $\mathrm{mm}$; range, -8.4 to $8.5 \mathrm{~mm}$ ) ( $\mathrm{p}<0.001$ ) (Table 1, Fig. 3).

The mean values of the possible influencing factors were: $51.5 \pm 6.3 \mathrm{~mm}$ (range, 36.9 to $69.6 \mathrm{~mm}$ ) for the F-OFF; $447.4 \pm 33.2$ $\mathrm{mm}$ (range, 397.0 to $498.2 \mathrm{~mm}$ ) for the $\mathrm{FL} ; 368.2 \pm 26.8 \mathrm{~mm}$ (range, 321.8 to $410.6 \mathrm{~mm}$ ) for the TL; $189.1 \pm 11.7 \mathrm{~mm}$ (range, 160.2 to $215.6 \mathrm{~mm}$ ) for the TW; $122.3 \pm 8.7 \mathrm{~mm}$ (range, 105.3 to 137.3 $\mathrm{mm}$ ) for the CW; $4.3^{\circ} \pm 1.3^{\circ}$ (range, $1.8^{\circ}$ to $7.1^{\circ}$ ) for the Rad-Q; and $1.5^{\circ} \pm 1.6^{\circ}$ (range, $-2.1^{\circ}$ to $4.1^{\circ}$ ) for the mechanical axis.

No correlation factor showed significant difference in the patellar tilt. However, radiological Q-angle showed a statistically significant correlation with the difference between the patellofemoral indices on weight-bearing and non-weight bearing positions, with regards to the lateral patellar subluxation $(\mathrm{p}=0.043$, $\gamma=0.094)$, LAT-PD $(\mathrm{p}=0.026, \gamma=0.112)$, and congruency angle $(\mathrm{p}<0.001, \gamma=0.488)$, obtained using univariate and multivariate regressing analysis (Table 2 ).

Table 1. Total Radiographic Results and Comparison of Radiographic Measurements between Standing and Supine Merchant Views

\begin{tabular}{|c|c|c|c|c|c|}
\hline Variable & Mean & $\mathrm{SD}$ & Min & Max & p-value \\
\hline Age (yr) & 27.77 & 6.75 & 18.00 & 39.00 & \\
\hline Standing patella TILT $\left({ }^{\circ}\right)$ & 3.66 & 2.00 & 0.10 & 8.50 & $<0.001$ \\
\hline Supine patella TILT $\left(^{\circ}\right)$ & 5.33 & 2.46 & 1.50 & 9.80 & \\
\hline Standing lateral $\mathrm{PF}\left({ }^{\circ}\right)$ & 11.30 & 4.73 & 1.00 & 20.90 & $<0.001$ \\
\hline Supine lateral PF $\left(^{\circ}\right)$ & 6.82 & 3.81 & 1.10 & 15.80 & \\
\hline Standing SUB-D (mm) & -1.96 & 1.80 & -5.80 & 4.60 & 0.004 \\
\hline Supine SUB-D (mm) & -1.04 & 2.75 & -6.60 & 4.60 & \\
\hline Standing lateral PD (mm) & -3.45 & 3.65 & -9.60 & 8.20 & $<0.001$ \\
\hline Supine lateral PD (mm) & -1.93 & 3.63 & -8.40 & 8.50 & \\
\hline Standing congruence $\left({ }^{\circ}\right)$ & -8.70 & 2.39 & -12.00 & -2.80 & $<0.001$ \\
\hline Supine congruence $\left({ }^{\circ}\right)$ & -3.53 & 4.51 & -11.00 & 11.20 & \\
\hline Femoral offset (mm) & 51.45 & 6.33 & 36.90 & 69.60 & \\
\hline Femoral length (mm) & 447.42 & 33.16 & 397.00 & 498.20 & \\
\hline Tibial length (mm) & 368.18 & 26.82 & 321.80 & 410.60 & \\
\hline Tibial width (mm) & 189.06 & 11.71 & 160.20 & 215.60 & \\
\hline Calf width (mm) & 122.25 & 8.65 & 105.30 & 137.30 & \\
\hline Radiographic $\mathrm{Q}$ angle $\left({ }^{\circ}\right)$ & 4.32 & 1.32 & 1.80 & 7.10 & \\
\hline Mechanical axis $\left({ }^{\circ}\right)$ & 1.49 & 1.56 & -2.10 & 4.10 & \\
\hline
\end{tabular}

TILT: tilt angle, PF: patellofemoral angle, SUB-D: subluxation distance, PD: patellar displacement. 

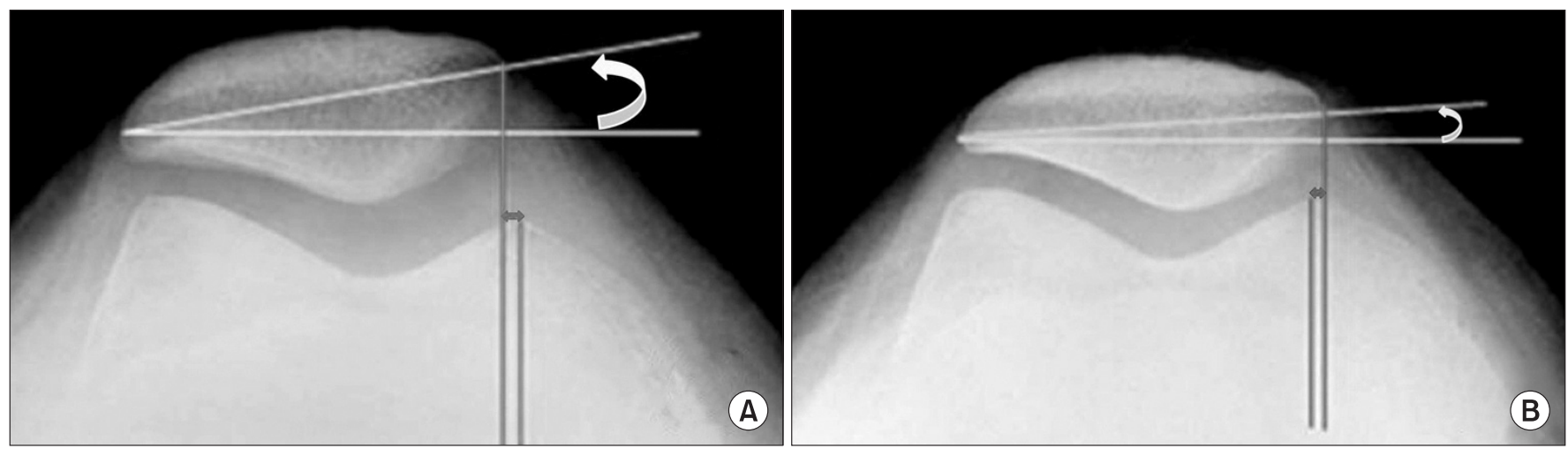

Fig. 3. (A) Supine Merchant view showing increased patellar tilt angle (white arrow) and lateral patellar displacement (grey arrow). (B) Standing (weight-bearing) Merchant view of the same patient, showing decreased patella tilt angle (white arrow) and lateral patellar displacement (grey arrow).

Table 2. Statistical Analysis of Correlation Factors Using Univariate and Mulitvariate Regression Analysis

\begin{tabular}{|c|c|c|c|c|}
\hline & \multicolumn{2}{|l|}{ Univariate } & \multicolumn{2}{|l|}{ Multivariate } \\
\hline & Factor (p-value) & R-square & Factor (p-value) & R-square \\
\hline Patella tilt & None & None & None & None \\
\hline Lateral patellofemoral angle & Femoral length (0.004) & 0.183 & Femoral length (0.038) & 0.257 \\
\hline Subluxation distance & Radiographic Q angle (0.043) & 0.094 & Radiographic Q angle (0.043) & 0.094 \\
\hline Lateral patellar displacement & Radiographic Q angle (0.026) & 0.112 & Radiographic Q angle (0.026) & 0.112 \\
\hline \multirow[t]{2}{*}{ Congruence angle } & Thigh width (0.004) & 0.220 & Thigh width (0.026) & 0.488 \\
\hline & Radiographic Q angle $(<0.001)$ & 0.340 & Radiographic Q angle $(<0.001)$ & 0.488 \\
\hline
\end{tabular}

\section{Discussion}

On the comparison between the supine- $\mathrm{M}$ and standing- $\mathrm{M}$ views, the TILT was decreased, the LAT-PF was increased, and the SUB-D and LAT-PD were decreased on the standing-M view. These results were consistent with our hypothesis that there would be a notable difference between the two views, suggesting the clinical significance of radiographic discrepancy in the assessment of the patellofemoral joint. In a recent study, Draper et al. ${ }^{4}$ reported that LAT-PD was significantly reduced under weightbearing conditions in patients with patellofemoral joint pain, although TILT did not exhibit significant changes ${ }^{4}$. Thus, it is our understanding that a diagnosis based on supine- $\mathrm{M}$ view alone could be inaccurate, eventually resulting in inappropriate treatment or unnecessary surgery.

Still, there is no agreement on the factors that may account for the difference under weight-bearing and non-weight-bearing conditions. It has been considered that complex interactions of various factors could be attributable to the discrepancy. Above all, the two most frequently encountered causes are the influence of the quadriceps femoris on the patella and the bone geometry of the patella and femur and patellofemoral joint motion during closed-chain contraction (weight-bearing condition).

Regarding the influence of the quadriceps femoris on the patella, the movement of the patella is mostly dependent on the quadriceps femoris. Considering that hip alignment and quadriceps femoris activation and strength vary during different movement conditions, weight-bearing could result in such radiographic changes in the patellofemoral joint ${ }^{4)}$. In addition, the onset of electromyographic (EMG) activity of the four components of the quadriceps femoris is more concurrent in closed-chain contraction than in open-chain isometric contraction. The onset of vastus medialis obliquus is significantly delayed in open-chain contraction and exhibits a lower amplitude of EMG than the other components ${ }^{15}$. This unbalanced onset of quadriceps activation could result in a lateral orientation of the patella in the spine position compared to the closed-chain contraction ${ }^{15)}$. Some previous studies showed that active quadriceps femoris contraction ${ }^{10)}$ and weight-bearing condition ${ }^{16)}$ could result in more lateral displacement and tilt of the patella than passive knee motion under nonweight-bearing condition. Although the exact weight-bearing condition was not reproduced in these studies, their results were 
similar to those measured on the weight- bearing radiographs in our study.

Regarding the bone geometry and joint motion, patellar kinematics is influenced by the axial and rotational alignment of the lower limb ${ }^{17,18}$. The normal knee function is related to pivoting in the medial and lateral sides of the knee and thus patellofemoral joint motion is affected by either the medial or lateral side pivoting $^{19)}$. It is well documented in the literature that knee joint motion under weight-bearing condition, or in closed-chain contraction, is different from that under non-weight-bearing condition. When the knee flexes with the foot on the floor, the femur is slightly externally rotated. Then, when the knee is completely extended, the femur is internally rotated and the knee joint is in its most stable position to support the body weight. Conversely, when the femur is fixated on a table with the foot off the ground, knee flexion results in internal rotation of the tibia whereas knee extension elicits external rotation of the tibia ${ }^{20-23)}$. Koo et al. ${ }^{19)}$ reported in a biomechanical study that the knee joint center of rotation is predominantly located on the lateral side and the femur is externally rotated and anteriorly translated relative to the tibia during normal walking (closed-chain contraction). In addition, knee joint motions are dependent on weight-bearing and nonweight-bearing activities: the center of rotation of the knee is in the medial compartment during a non-weight-bearing activity, whereas it is shifted laterally during a weight-bearing activity ${ }^{24)}$. Recent studies demonstrated that internal rotation of the tibia is correlated with sliding of the joint on the medial compartment and lateral pivoting occurs during a weight-bearing activity ${ }^{19,25)}$. Limitations of our study include that the patellofemoral joint was not assessed during normal ambulation and the patients had no patellofemoral pain. We believe that the influence of weight-bearing on radiographic changes of the patellofemoral joint should be confirmed in further studies involving patients with patellofemoral joint pain to establish the clinical significance of our study.

\section{Conclusions}

The standing- $M$ view showed increased medial tilt of the patella, decreased lateral displacement of the patella and increased congruence compared to those on the supine-M view. The FL and Rad-Q were the factors that influenced such radiographic discrepancy. This study showed that weight-bearing conditions have an influence on the patellofemoral joint indices. Therefore, we believe that radiographic assessment of the patellofemoral joint should be based on both the supine-M and standing- $\mathrm{M}$ views.

\section{Conflict of Interest}

No potential conflict of interest relevant to this article was reported.

\section{References}

1. Fulkerson JP. Diagnosis and treatment of patients with patellofemoral pain. Am J Sports Med. 2002;30:447-56.

2. DeHaven KE, Lintner DM. Athletic injuries: comparison by age, sport, and gender. Am J Sports Med. 1986;14:218-24.

3. Fredericson M, Yoon K. Physical examination and patellofemoral pain syndrome. Am J Phys Med Rehabil. 2006;85:234-43.

4. Draper CE, Besier TF, Fredericson M, Santos JM, Beaupre GS, Delp SL, Gold GE. Differences in patellofemoral kinematics between weight-bearing and non-weight-bearing conditions in patients with patellofemoral pain. J Orthop Res. 2011;29:312-7.

5. Baldini A, Anderson JA, Cerulli-Mariani P, Kalyvas J, Pavlov H, Sculco TP. Patellofemoral evaluation after total knee arthroplasty. Validation of a new weight-bearing axial radiographic view. J Bone Joint Surg Am. 2007;89:1810-7.

6. Nha KW, Papannagari R, Gill TJ, Van de Velde SK, Freiberg AA, Rubash HE, Li G. In vivo patellar tracking: clinical motions and patellofemoral indices. J Orthop Res. 2008;26:1067-74.

7. Noehren B, Barrance PJ, Pohl MP, Davis IS. A comparison of tibiofemoral and patellofemoral alignment during a neutral and valgus single leg squat: an MRI study. Knee. 2012;19:380-6.

8. Draper CE, Besier TF, Santos JM, Jennings F, Fredericson M, Gold GE, Beaupre GS, Delp SL. Using real-time MRI to quantify altered joint kinematics in subjects with patellofemoral pain and to evaluate the effects of a patellar brace or sleeve on joint motion. J Orthop Res. 2009;27:571-7.

9. Wilson NA, Press JM, Koh JL, Hendrix RW, Zhang LQ. In vivo noninvasive evaluation of abnormal patellar tracking during squatting in patients with patellofemoral pain. J Bone Joint Surg Am. 2009;91:558-66.

10. Brossmann J, Muhle C, Schroder C, Melchert UH, Bull CC, Spielmann RP, Heller M. Patellar tracking patterns during active and passive knee extension: evaluation with motiontriggered cine MR imaging. Radiology. 1993;187:205-12.

11. Powers CM, Ward SR, Fredericson M, Guillet M, Shellock FG. Patellofemoral kinematics during weight-bearing and 
non-weight-bearing knee extension in persons with lateral subluxation of the patella: a preliminary study. J Orthop Sports Phys Ther. 2003;33:677-85.

12. McWalter EJ, Hunter DJ, Wilson DR. The effect of load magnitude on three-dimensional patellar kinematics in vivo. J Biomech. 2010;43:1890-7.

13. Fukui N, Nakagawa T, Murakami S, Hiraoka H, Nakamura K. A modified system of stress radiography for patellofemoral instability. J Bone Joint Surg Br. 2003;85:1128-33.

14. Grelsamer RP, Weinstein CH, Gould J, Dubey A. Patellar tilt: the physical examination correlates with MR imaging. Knee. 2008;15:3-8.

15. Stensdotter AK, Hodges PW, Mellor R, Sundelin G, HagerRoss C. Quadriceps activation in closed and in open kinetic chain exercise. Med Sci Sports Exerc. 2003;35:2043-7.

16. Shellock FG, Mink JH, Deutsch AL, Foo TK, Sullenberger P. Patellofemoral joint: identification of abnormalities with active-movement, "unloaded" versus "loaded" kinematic MR imaging techniques. Radiology. 1993;188:575-8.

17. Post WR, Teitge R, Amis A. Patellofemoral malalignment: looking beyond the viewbox. Clin Sports Med. 2002;21:52146 ,

18. Lee TQ, Morris G, Csintalan RP. The influence of tibial and femoral rotation on patellofemoral contact area and pres- sure. J Orthop Sports Phys Ther. 2003;33:686-93.

19. Koo S, Andriacchi TP. The knee joint center of rotation is predominantly on the lateral side during normal walking. J Biomech. 2008;41:1269-73.

20. Blaha JD, Mancinelli CA, Simons WH, Kish VL, Thyagarajan $\mathrm{G}$. Kinematics of the human knee using an open chain cadaver model. Clin Orthop Relat Res. 2003;(410):25-34.

21. Dennis DA, Mahfouz MR, Komistek RD, Hoff W. In vivo determination of normal and anterior cruciate ligamentdeficient knee kinematics. J Biomech. 2005;38:241-53.

22. Freeman MA, Pinskerova V. The movement of the normal tibio-femoral joint. J Biomech. 2005;38:197-208.

23. Iwaki H, Pinskerova V, Freeman MA. Tibiofemoral movement 1: the shapes and relative movements of the femur and tibia in the unloaded cadaver knee. J Bone Joint Surg Br. 2000;82:1189-95.

24. Dyrby CO, Andriacchi TP. Secondary motions of the knee during weight bearing and non-weight bearing activities. J Orthop Res. 2004;22:794-800.

25. Hoshino $\mathrm{Y}$, Tashman $\mathrm{S}$. Internal tibial rotation during in vivo, dynamic activity induces greater sliding of tibio-femoral joint contact on the medial compartment. Knee Surg Sports Traumatol Arthrosc. 2012;20:1268-75. 